\title{
Computationally Efficient Filtering Algorithms for Engine Torque Estimation
}

\author{
Alexander Stotsky and Ilya Kolmanovsky
}

\begin{abstract}
A new computationally efficient filtering algorithm for reconstruction of the first harmonic of a periodic signal is presented. The algorithm allows to recover the combustion quality information from the engine speed measurements which are noise contaminated. The algorithm is applied to the torque estimation problem for a $V 8$ spark ignition engine.
\end{abstract}

\section{INTRODUCTION}

The performance of engine torque estimation affects directly a drivability of the vehicle. The engine torque estimation function is based on monitoring of the cylinder individual fluctuations of the high resolution engine speed signal (see references [3], [8], [9]). The engine speed signal is based on the measurements of a passage time between two subsequent teeth on a crankwheel. The passage time decreases as the rotational speed increases thus the time interval errors increase. Moreover, low frequency oscillations from the powertrain and high frequency oscillations due to the crankshaft torsion, together with vibrations induced by the road, act as disturbances on the crankshaft. These disturbances influence directly the performance of the engine speed signal and consequently the torque monitoring function. The problem described above is more important for six and eight cylinder engines than for five cylinder engines. This is due to a larger amount of combustion events which should be recognized in the presence of the described disturbances.

This necessitates the development of the computationally efficient filtering algorithms which recover the engine speed fluctuations corresponding to the combustion events from the noise contaminated measurements.

The approach proposed in this paper is based on the trigonometric interpolation of the measured engine speed data in the least squares sense. In application of the idea of the window moving in time (see references [2], [4]) to numerical differentiation by using polynomial of a certain order, the trigonometric polynomial is fitted here to the measured data. The trigonometric interpolation is fairly suitable for the engine speed approximation due to the periodic nature of both engine rotational dynamics and combustion forces as functions of a crank angle. The filtering technique uses the periodic signal at the combustion frequency and the amplitudes of the trigonometric functions

\footnotetext{
A. Stotsky (corresponding author) is with Volvo Car Corporation, Engine Design and Development, Dept. 97542, HA1N, SE- 40531 Gothenburg, Sweden. Email: astotsky@volvocars.com. The work of this author was done within the Volvo $6-\sigma$ programme.

I.V. Kolmanovsky is with Ford Motor Company, 2101 Village Road,

Dearborn, MI 48124. Email: ikolmano@ford.com.
}

are updated according to the trigonometric interpolation method. Applying the idea of the recursive computations to the moving window described in [10], the computationally efficient recursive algorithms are developed in this case for the trigonometric polynomials.

A Volvo XC90 passenger car equipped with $V 8$ engine was used in the experiments. Algorithms are implemented in MATLAB $^{1}$ and applied to the measured data collected from the experimental vehicle.

The paper is organized as follows. The next section is devoted to the problem statement. The third section is devoted to the recursive trigonometric interpolation method and its application to the engine torque estimation. The fourth section contains brief concluding remarks.

\section{Problem Statement}

As a rule, a passage time between two teeth on a crankwheel is measured in production engines. The high resolution engine speed signal is then calculated via the elapsed time by using the first difference method.

The combustion state of the given cylinder is defined via the amplitude. The amplitude for the cylinder, whose power stroke occurs in the interval, in turn is defined as the difference between maximal and minimal values of the high resolution engine speed signal. The corresponding amplitude, which is the measure of the crankwheel speed perturbations induced by the periodic impulsive cylinder individual torque contributions, provides a mean for estimation of the engine torque [5]. Here and below, a nonstandard definition is used, and under the term 'amplitude' the difference between maximal and minimal values is understood.

Figure 1 shows the harmonics of the engine speed signal at $1800 \mathrm{rpm}$ and $5400 \mathrm{rpm}$ calculated by the Discrete Fourier Transform (DFT) method [6]. The input sequence was sampled with the step $30 \mathrm{CA}$ ( Crank Angle ) degrees and the data was acquired over a $720 \mathrm{CA}$ degree window. Amplitudes are plotted as a function of a harmonic number of a periodic signal with the period of 720 CA ( Crank Angle ) degrees. The harmonic number is defined as an integer which is equal to the ratio of two periods, $n_{h}=$ $\frac{720^{\circ}}{T_{h}}$, where $T_{h}$ is the period of the harmonic. Figure 1 shows that the engine speed signal at low rotational speeds has a dominating component which corresponds to the combustions events. The engine speed signal at high rotational speeds has fluctuations which occur as a consequence of

\footnotetext{
${ }^{1}$ MATLAB is a registered mark of the Mathworks, Inc of Natick, MA.
} 
the combustion events, low frequency oscillations from the powertrain as well as high frequency oscillations due to the crankshaft torsion. The high frequency oscillations due to the crankshaft twist and low frequency oscillations from the powertrain could be greater than the oscillations induced by the combustion events. Notice that, the input sequence was sampled with the step $30^{\circ}$ which is a relatively low rate sampling. At this rate the high order harmonic components could be aliased within the range of a lower frequencies. Thus, the higher amplitudes at the lower harmonics $\left(n_{h}<8\right.$ ) showed in Fig.1, could be the superposition of low frequency torsional oscillations and the aliased high frequency oscillations.

The problem stated in this paper is to design the computationally efficient algorithms which recover the engine speed fluctuations corresponding to the combustion events from the noise contaminated measurements of the engine speed.

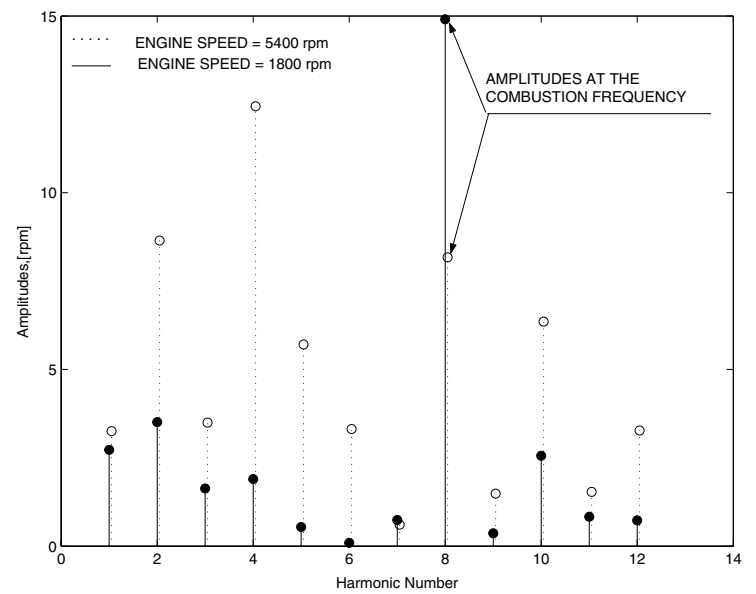

Figure 1:

Harmonic contents of the engine speed signal at $1800 \mathrm{rpm}$ and $5400 \mathrm{rpm}$ computed via the DFT method. The input sequence is sampled with the step $30^{\circ}$. The data is acquired over $720^{\circ}$ window. The measurements are made on the experimental vehicle on the chassis rolls.

Amplitudes are plotted as a function of the harmonic number of the signal with the period of $720 \mathrm{CA}$ degrees. The engine is operating at 50\% load. Amplitudes at 1800 rpm are plotted with solid line and amplitudes at 5400 rpm are plotted with the dotted line.

\section{RECURSIVE TRIGONOMETRIC INTERPOLATION METHOD}

\section{A. General Description}

Suppose that there is a set of the Crank Angle synchronized data $y_{l}, l=1, \ldots, w$, measured at the following points:

$$
x_{1}=\Delta, \quad x_{2}=2 \Delta, \quad \ldots . x_{w}=w \Delta, \quad \Delta=\frac{360^{0}}{w},
$$

where $\Delta$ is a step size. For example, the high resolution engine speed signal is sampled on the basis of a Crank Angle (CA) with the step $\Delta$, namely,

$$
\Delta=\frac{L_{c}}{n_{p} N}
$$

where $\Delta$ is the discretization step ( CA degrees ), $N$ is the number of the cylinders of the engine, $n_{p}$ is the number of points measured for each combustion event ( $n_{p} \geq 3$ ), $L_{c}$ is the length of the engine cycle in CA degrees ( as a rule, $L_{c}=720^{\circ}$ ). Since an engine crankshaft is usually provided with 58 teeth and a gap corresponding to two missing teeth, the step $\Delta$ should be a multiple of $6^{\circ}$.

Assume that the number of measurements $w$ is greater than or equal to $(2 n+1)$, where $n$ is the number of the frequencies of the signal. Then there exists the trigonometric polynomial in the following form:

$$
T_{n}(x)=a_{0}+\sum_{q=1}^{n}\left(a_{q} \cos (q x)+b_{q} \sin (q x)\right),
$$

which gives the best approximation of the measured data $y_{l}$ at the points $x_{l}$ in the least-squares sense. Notice, that the frequencies $q=1,2, \ldots, n$ defined in (3) should include the combustion frequency, as well as low powertrain frequencies and high frequencies due to the crankshaft torsion.

Measured data should be approximated by the polynomial (3) in the least squares sense. The error to be minimized at every step is as follows:

$$
E=\sum_{l=1}^{w}\left(y_{l}-\left(a_{0}+\sum_{q=1}^{n}\left(a_{q} \cos \left(q x_{l}\right)+b_{q} \sin \left(q x_{l}\right)\right)\right)\right)^{2} \text {. }
$$

Straightforward calculations show that the coefficients which minimize (4) are the following:

$$
\begin{aligned}
a_{0} & =\frac{1}{w} \sum_{l=1}^{w} y_{l}, \\
a_{q} & =\frac{2}{w} \sum_{l=1}^{w} y_{l} \cos \left(q x_{l}\right), \\
b_{q} & =\frac{2}{w} \sum_{l=1}^{w} y_{l} \sin \left(q x_{l}\right) .
\end{aligned}
$$

The expressions (3), (5) - (7) are known as the DFT method [6]. The coefficients (5), (6), (7) of the DFT have such a simple form due to the property of the orthogonality of the trigonometric polynomials in the interval $\left[0 \div 360^{\circ}\right)$. It is interesting to note that the trigonometric polynomial can be fitted to the measured data at any interval. However, for computation of the coefficients $a_{0}, a_{q}, b_{q}$ the matrix inversion is required, as it is usual for the least-squares fitting. Therefore the DFT method can be seen as a special case of the trigonometric interpolation method.

The main result of this paper is formulated below. 
B. Introduction of a moving window and recursive algorithms

The amount of computations in (5) - (7) can be reduced by introduction of the local fixed coordinates, i.e., moving window of a size $w$. The same idea was proposed in the spline interpolation method described in [4] and is based on the on-line least-squares polynomial fitting over the window moving in time. The idea of the window moving in time is illustrated in Figure 2. The window is defined in the form of the local coordinates $X_{L}, Y_{L}$. Then, the least squares curve fitting problem is solved in local coordinates and the result is transformed to the original coordinate system. Moreover, at every step the coefficients (5) - (7) can be computed recursively using the information from the previous step. This makes the whole scheme computationally efficient and implementable.

Consider one step of the window moving in time. Assume that there is a set of measurements at step $(k-1)$, $\{y(1), \quad y(2), \quad y(3), \quad \ldots, \quad y(w)\}$, which is measured in the following local coordinate system based on the crank angle $\{0, \quad \Delta, 2 \Delta, \quad \ldots, \quad(w-1) \Delta\}$, where $\Delta$ is defined by (2). At step $k$, new value $y(w+1)$ enters the window while $y(1)$ leaves the window. Whence, at step $k$ there is a set of measurements $\{\quad y(2), \quad y(3), \quad y(4)$,

$y(w+1)\}$, measured in the same local coordinate system. One step of the moving window is shown in Figure 2, where the engine speed is measured for the $V 8$ engine with the step $\Delta=30^{\circ}$.

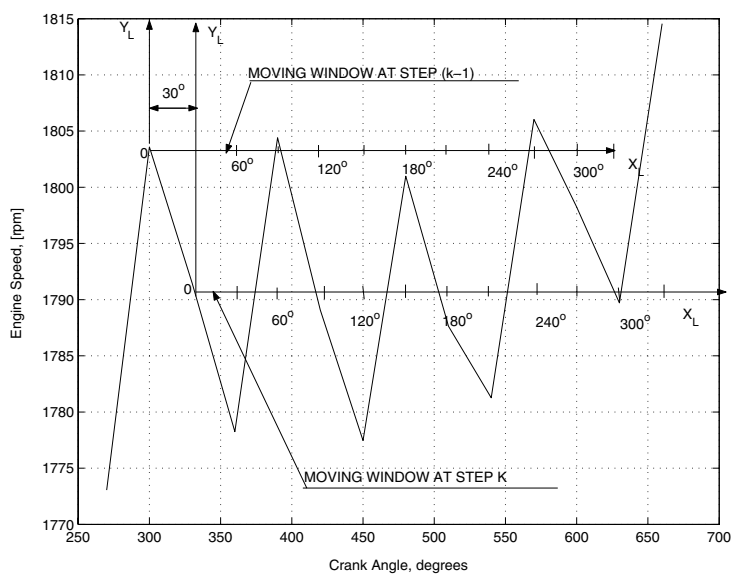

Figure 2

Measurements with the step $30 \mathrm{CA}$ degrees on the V8 engine. The engine speed is plotted with the solid line. The engine is operating at full load. Window of the size $w=12$ moving in time is defined in the form of local coordinates $X_{L}, Y_{L}$.

The coefficients $(5)-(7)$ at step $(k-1)$ are defined as follows:

$$
a_{0(k-1)}=\frac{1}{w} \sum_{i=1}^{w} y(i),
$$

$$
\begin{aligned}
a_{q(k-1)} & =\frac{2}{w} \sum_{i=1}^{w} y(i) \cos ((i-1) q \Delta), \\
b_{q(k-1)} & =\frac{2}{w} \sum_{i=1}^{w} y(i) \sin ((i-1) q \Delta),
\end{aligned}
$$

where $q$ denotes the frequency. The coefficients (8) - (10) at step $k$ are defined as follows:

$$
\begin{aligned}
a_{0 k} & =\frac{1}{w} \sum_{i=2}^{w+1} y(i), \\
a_{q k} & =\frac{2}{w} \sum_{i=2}^{w+1} y(i) \cos ((i-2) q \Delta), \\
b_{q k} & =\frac{2}{w} \sum_{i=2}^{w+1} y(i) \sin ((i-2) q \Delta) .
\end{aligned}
$$

The coefficients (11) - (13) are to be expressed via the coefficients (8) - (10).

Straightforward calculations give the expression for the first coefficient,

$$
a_{0 k}=a_{0(k-1)}+c_{q(k-1)}
$$

where $c_{q(k-1)}=\frac{1}{w}(y(w+1)-y(1))$, simply meaning that the value $y(w+1)$ enters the window, while the value $y(1)$ leaves the window.

Starting with $a_{q k}$, one gets,

$$
\begin{aligned}
a_{q k} & =\frac{2}{w} \sum_{i=2}^{w+1} y(i) \cos ((i-2) q \Delta) \\
& =\frac{2}{w} y(w+1) \cos ((w-1) q \Delta) \\
& +\frac{2}{w} \sum_{i=2}^{w} y(i) \cos ((i-1) q \Delta-q \Delta)= \\
& \frac{2}{w} y(w+1) \cos ((w-1) q \Delta) \\
& +\cos (q \Delta)\left(a_{q(k-1)}-\frac{2}{w} y(1)\right) \\
& =b_{q(k-1)} \sin (q \Delta) b_{q(k-1)}(q)+d_{q(k-1)} \cos (q \Delta),
\end{aligned}
$$

and

$$
\begin{aligned}
b_{q k} & =\frac{2}{w} \sum_{i=2}^{w+1} y(i) \sin ((i-2) q \Delta) \\
& =\frac{2}{w} y(w+1) \sin ((w-1) q \Delta) \\
& +\frac{2}{w} \sum_{i=2}^{w} y(i) \sin ((i-1) q \Delta-q \Delta)= \\
& \frac{2}{w} y(w+1) \sin ((w-1) q \Delta) \\
+ & \cos (q \Delta)\left(b_{q(k-1)}-\frac{2}{w} y(2) \sin (q \Delta)\right)
\end{aligned}
$$




$$
\begin{aligned}
& -\quad \sin (q \Delta)\left(a_{q(k-1)}-\frac{2}{w}(y(1)\right. \\
& +y(2) \cos (q \Delta)) \\
& =-d_{q(k-1)} \sin (q \Delta)+b_{q(k-1)} \cos (q \Delta),
\end{aligned}
$$

where $d_{q(k-1)}=a_{q(k-1)}+2 c_{q(k-1)}$. Notice that (15), (16) can be expressed in a single matrix equation

$$
s_{q k}=A_{q k} z_{q},
$$

where

$$
\begin{gathered}
s_{q k}=\left(\begin{array}{c}
a_{q k} \\
b_{q k}
\end{array}\right) \\
A_{q k}=\left(\begin{array}{cc}
b_{q(k-1)} & d_{q(k-1)} \\
-d_{q(k-1)} & a_{q(k-1)}
\end{array}\right),
\end{gathered}
$$

is a skew symmetric matrix and

$$
z_{q}=\left(\begin{array}{c}
\sin (q \Delta) \\
\cos (q \Delta)
\end{array}\right)
$$

is a constant vector. Implementation of the algorithms (14) - (16) requires 5 multiplications and 7 additions only at a single frequency. The algorithms are suitable for implementation on a simple controller that has a multiply and add processor.

The number of the multiplications for the DFT computation is proportional to the number of the samples of the input sequence ( the size of the moving window $w$ ) at a single frequency. The coefficients $a_{0 k}, a_{q k}$ and $b_{q k}$ of the scheme proposed here are computed via (8) - (10) at the first step. Hence the number of the arithmetical operations which is required for implementation of the algorithms proposed here and the DFT algorithms is the same at the first step. However, the number of the arithmetical operations for recursive algorithms (14),(15) and (16) does not depend on the window size $w$ at the subsequent steps. Then the advantage of the method proposed here with respect to the DFT method increases with the window size $w$.

The algorithms proposed above can also be used in the case of the engine speed transients, provided that a "slowly" varying trend of the engine speed is properly compensated. A simple compensation technique is described below. The measured engine speed signal $\omega_{k}$ is filtered by the following low pass filter:

$$
\omega_{f k}=\omega_{f(k-1)}-\frac{\Delta t}{\tau_{f}\left(\omega_{k}, \dot{\omega}_{k}\right)}\left(\omega_{f(k-1)}-\omega_{k}\right),
$$

where $\omega_{f k}$ is a filtered engine speed and $\tau_{f}\left(\omega_{k}, \dot{\omega}_{k}\right)$ is a "time constant" of the filter (18), $\Delta t$ [sec] is the discretization step. The time constant is realized as a look-up table with engine speed $\omega_{k}$ and its derivative $\dot{\omega}_{k}$, which is estimated by the first difference method as two inputs. The look-up table is calibrated so that the time constant is reduced under the transients to capture fast changes in engine speed, and is increased under the steady-state conditions. The following difference,

$$
y_{k}=\omega_{k}-\omega_{f k}
$$

between measured engine speed signal $w_{k}$ and filtered signal $\omega_{f k}$ represents the fluctuations which occur as a consequence of the combustion events, contaminated with errors. This difference is approximated by the trigonometric interpolation method. Then the engine speed is approximated via the sum of two components. The first one is the filtered engine speed $\omega_{f k}$ which approximates "slowly" varying trend of the engine speed. The second one is the approximation of the combustion events.

The variable $y_{k}$ is approximated by $\hat{y}_{k}$ according to the following equation:

$\hat{y}_{k}=a_{0 k}+\sum_{q=1}^{n}\left(a_{q k} \cos (q(w-1) \Delta)+b_{q k} \sin (q(w-1) \Delta)\right)$,

where the coefficients are updated as follows:

$$
\begin{aligned}
& a_{0 k}=a_{0(k-1)}+c_{q(k-1)}, \\
& a_{q k}=b_{q(k-1)} \sin (q \Delta)+d_{q(k-1)} \cos (q \Delta), \\
& b_{q k}=-d_{q(k-1)} \sin (q \Delta)+b_{q(k-1)} \cos (q \Delta),
\end{aligned}
$$

where $d_{q(k-1)}=a_{q(k-1)}+2 c_{q(k-1)}$ and $c_{q(k-1)}=$ $\frac{1}{w}(y(w+1)-y(1))$. The value of the interpolating polynomial $\hat{y}_{k}$ is taken at the end of the moving window $(w-1) \Delta$. Then the engine speed can be approximated as follows:

$$
\hat{\omega}_{k}=\omega_{f k}+\hat{y}_{k}
$$

where $\hat{y}_{k}$ is computed via (20) - (23).

Further in the paper, the trigonometric interpolation is used as a filter at the combustion frequency, i.e.,

$$
\begin{aligned}
\hat{y}_{c k} & =a_{0 k}+a_{q_{c} k} \cos \left(q_{c}(w-1) \Delta\right) \\
& +b_{q_{c} k} \sin \left(q_{c}(w-1) \Delta\right), \\
\hat{\omega}_{c k} & =\omega_{f k}+\hat{y}_{c k},
\end{aligned}
$$

where $q_{c}=\frac{N \pi}{360}$ is the combustion frequency, and $N$ is the number of the engine cylinders. The combustion frequency is the frequency of the signal whose period is $\frac{720^{\circ}}{N}$. The coefficients $a_{0 k}, a_{q c k}, b_{q c k}$ are computed according to the recursive formulae (21) - (23). Figure 3 shows the result of the filtering at the firing frequency of the engine speed signal by the filter (25), (26). It can be seen that the amplitude information is recovered on the signal which is filtered by the filter (25), (26). 


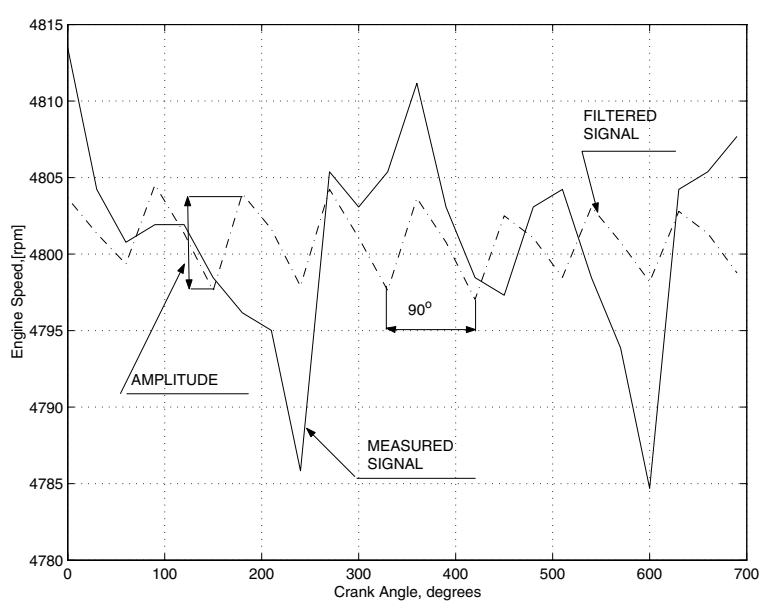

Figure 3

Measurements with the step $30 \mathrm{CA}$ degrees on the V8 engine. A single engine cycle is plotted. The engine speed

is plotted with the solid line. Relative load is $45 \%$.

Filtered signal corresponding to the firing frequency is plotted with dashdot line. The size of the moving window is equal to twelve $(w=12)$.

\section{Application of the recursive trigonometric interpolation method to the estimation of the engine torque}

The filtered engine speed signal can now be used for engine torque estimation. The time rate change of the crankshaft angular velocity is proportional to the torque acting on the system. The ideal engine rotational dynamics can be described as follows:

$$
J \dot{\omega}(t)=T_{e}-T_{l},
$$

where $\omega$ is the speed of the engine, $J$ is the crankshaft inertia, $T_{e}$ is the brake torque of the engine, and $T_{l}$ is the engine load torque. Equation (27) can be transformed into the crank angle domain using the chain rule of differentiation and employing the crank angle $\theta$ as an independent variable,

$$
\omega J \frac{d \omega}{d \theta}=T_{e}-T_{l}
$$

Integrating over a crank angle interval $\left[\begin{array}{ll}\theta_{s} & \theta_{f}\end{array}\right]$ (e.g., $\theta_{s}$ and $\theta_{f}$ may be defined so that the engine speed assumes a minimal value at $\theta_{s}$ and maximum value at $\theta_{f}$ ), we obtain

$$
\omega\left(\theta_{f}\right)-\omega\left(\theta_{s}\right)=\frac{1}{J} \int_{\theta_{s}}^{\theta_{f}} \frac{\left(T_{e}-T_{l}\right)}{\omega} d \vartheta .
$$

Since the torque is a continuous function in the interval $\left[\theta_{s}, \theta_{f}\right]$, there exists $\theta_{1} \in\left[\theta_{s}, \theta_{f}\right]$, such that the following holds:

$$
\int_{\theta_{s}}^{\theta_{f}} \frac{\left(T_{e}-T_{l}\right)}{\omega} d \vartheta=\frac{\left(T_{e}\left(\theta_{1}\right)-T_{l}\left(\theta_{1}\right)\right)\left(\theta_{f}-\theta_{s}\right)}{\omega\left(\theta_{1}\right)} .
$$

Substituting (30) into (29) and with the amplitude, $A_{i}$, defined for the $i$ th engine cylinder, $i=1, \cdots, N$, as

$$
A_{i}=\omega\left(\theta_{f}\right)-\omega\left(\theta_{s}\right)
$$

we obtain

$$
A_{i}=\frac{\left(\theta_{f}-\theta_{s}\right)}{J \omega\left(\theta_{1}\right)}\left(T_{e}\left(\theta_{1}\right)-T_{l}\left(\theta_{1}\right)\right)
$$

Since

$$
\frac{1}{\omega\left(\theta_{1}\right)} \approx \frac{1}{\bar{\omega}}-\frac{1}{\bar{\omega}^{2}}\left(\omega\left(\theta_{1}\right)-\bar{\omega}\right)
$$

where $\bar{\omega}$ is the mean value of the engine speed in the interval, and since $\frac{1}{\bar{\omega}^{2}}$ is small and can be neglected, it follows that

$$
A_{i} \approx \frac{\left(\theta_{f}-\theta_{s}\right)}{J \bar{\omega}}\left(T_{e}\left(\theta_{1}\right)-T_{l}\left(\theta_{1}\right)\right)
$$

Thus $A_{i}$ can be viewed as a measure of the difference between the engine brake torque and the load torque. The expression (33) may be suitable for estimating $T_{e}\left(\theta_{1}\right)$ in transient conditions if the load torque, $T_{l}\left(\theta_{1}\right)$, is known. Unfortunately, the load torque depends on vehicle mass and road grade which are unknown parameters. See reference [11] for challenges and methods to simultaneously estimate the vehicle mass and the road grade. The load torque may be estimated using the automatic transmission torque converter turbine speed measurement, if the torque converter is in the unlocked state, or using a wheel speed measurement.

More progress can be made for close to steady-state or slow transient conditions. Let $\bar{T}_{e}$ denote the mean value of the $i$ th cylinder torque, i.e.,

$$
T_{e}(\theta)=\bar{T}_{e}+\tilde{T}_{e}(\theta),
$$

where $\tilde{T}_{e}(\theta)$ is the torque fluctuation.

The torque of each cylinder can be presented as a sum of two components, namely the mean value of the torque and the torque fluctuations. The Dual Mass Flywheel (DMF) which is widely used in production vehicles [1] reduces the crankshaft oscillations transmitted to the powertrain, offering the vibration isolation and acting like a lowpass filter. Then the torque which is measured on the gear shaft is the mean value of the engine brake torque, which in turn is estimated via the torque fluctuations on the flywheel. In other words, the periodic flywheel speed variations induced by the periodic torque contributions of individual cylinders are isolated due to the DMF from the rest of the driveline, providing a mean for the torque estimation. Due to DMF in the driveline it can be assumed that in close to steady-state or in slow transient conditions,

$$
T_{l}\left(\theta_{1}\right)=\bar{T}_{e}
$$

Then, (33) implies

$$
A_{i} \approx \frac{\left(\theta_{f}-\theta_{s}\right)}{J \bar{\omega}} \tilde{T}_{e}\left(\theta_{1}\right) .
$$

An increase or decrease in $\tilde{T}_{e}$ correlates with, respectively, an increase or decrease in $\bar{T}_{e}$ [5]. A simple, intuitive way to understand this is to note that $\bar{T}_{e}$ increases almost linearly 
with the increase in the quantity of fuel while with the appropriate $^{2}$ choice of $\theta_{s}, \theta_{f}$ the cylinder pressure rise during the cycle and the cylinder torque will also exhibit a close to linear trend with the increase in fuel quantity. Thus we find that $\bar{T}_{e}$, the mean-value of the $i$ th engine cylinder torque, can be correlated to $A_{i}$. If the cylinders are balanced and the engine is in steady-state conditions, the mean value of the overall engine torque can be correlated to the average, $\bar{A}$, of fluctuations $A_{i}$ over a certain number of combustion events. Usually, averaging over $10-15$ events is sufficient. As a result, a function $f(\cdot)$ can be developed that predicts engine torque as a function of engine speed, $\bar{\omega}$, and $\bar{A}$, i.e.,

$$
\bar{T}_{e}=f(\bar{\omega}, \bar{A}) .
$$

This function can be implemented as a 2D look-up table and calibrated on an engine in a vehicle equipped with a torque sensor at the flywheel for the duration of the calibration phase. Although the look-up table (34) may, in principle, have to be replicated for different gear ratios (due to changing inertia with changing gear), experimental evidence from our particular vehicle suggests that the gear dependence is not significant.

Figure 4 illustrates the verification of the proposed algorithm. The torque measured on a rig (chassis rolls) via the measurements of the traction force is plotted with the solid line and the estimated torque obtained by using the technique proposed above is plotted with a dotted line. Figure 3 confirms a good correlation between measured and estimated engine brake torques.

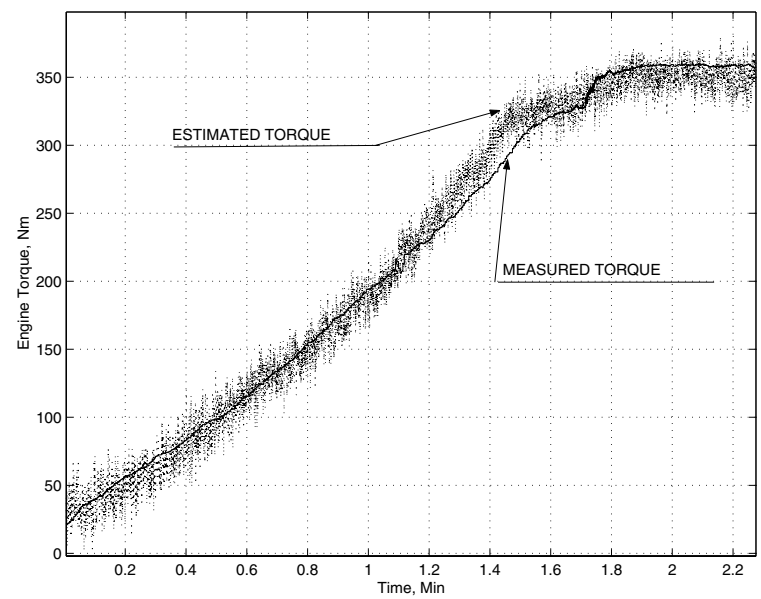

Figure 4

Measurements from the V8 engine. The engine speed is $4800 \mathrm{rpm}$. Measured engine torque is plotted with solid line. Estimated torque is plotted with dotted line.

\section{CONCLUSION}

It has been shown that the torque estimation technique can benefit from the availablity of the combustion quality information provided by the algorithm proposed in this paper.

\footnotetext{
${ }^{2}$ Reference [7] discusses how the window $\left[\theta_{s}, \theta_{f}\right]$ can be optimally selected to improve individual cylinder torque observability.
}

A new computationally efficient algorithm which provides filtering at the engine firing frequency was designed and verified.

\section{REFERENCES}

[1] Albers A., Albrecht M., Kruger A., Lux R. (1997). New Methodology for Power Train Development in the Automotive Engineering Integration of Simulation, Design and Testing, SAE Paper 2001-013303.

[2] Dabroom A., Khalil H. (1999). Discrete-Time Implementation of High-Gain Observers for Numerical Differentiation, International Journal of Control 72 (17), p.p. 1523-1527.

[3] Ginoux S., Champoussin J.C. (1997). Engine Torque Determination by Crankangle Measurements: State of the Art, Future Prospects , SAE Paper 970532 .

[4] Diop S., Grizzle J., Moraal P., Stefanopoulou A. (1994). Interpolation and Numerical Differentiation for Observer Design, Proc. American Control Conference, Baltimore, Maryland, p.p. 1329 - 1333.

[5] Kay, I.W., and Lehrach, R.P.C. Torque Sensing for Controlled Alternative-Fuel Combustion in Diesel Engines. SAE Paper 841007.

[6] Lyons R. Understanding Digital Signal Processing, Addison Wesley, 1997.

[7] van Nieuwstadt, M., and Kolmanovsky, I. Detecting and Correcting Cylinder Imbalance in Direct Injection Engines. Journal of Dynamic Systems, Measurement and Control, vol. 123, September 2001, pp. 413-424, 2001.

[8] Rizzoni G. (1989) Estimate of Indicated Torque from Crankshaft Speed Fluctuations: A Model for the Dynamics of the IC Engine, IEEE Transactions on Vehicular Technology, vol. 38, no. 3, pp. 168179.

[9] Rizzoni G., Guezennec Y., Soliman A. (2003). Engine Control Using Torque Estimation US Patent Application Publication 2003/0167118 Al.

[10] Stotsky A., Forgo A. (2004). Recursive Spline Interpolation Method for Real Time Engine Control Applications, Control Engineering Practice, vol.12, pp.409-416.

[11] Vahidi, A., Druzhinina, M., Stefanopoulou, A., and Peng, H. Simultaneous mass and time-varying grade estimation for heavy-duty vehicles. Proceedings of 2003 American Control Conference, Denver, Colorado, 4951-4956, 2003. 